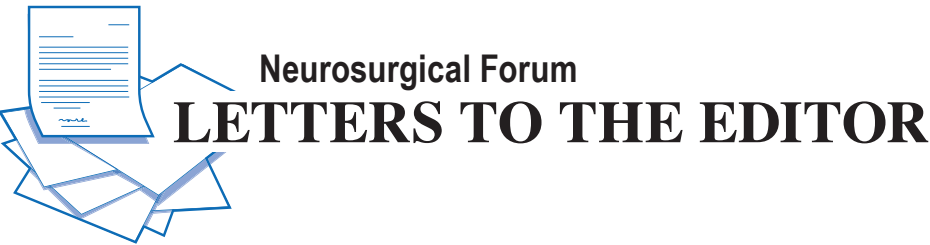

\section{Healthy competition}

TO THE EDITOR: We read with great interest the article by Wadhwa et al. ${ }^{1}$ (Wadhwa H, Shah SS, Shan J, et al. The neurosurgery applicant's "arms race": analysis of medical student publication in the Neurosurgery Residency Match. J Neurosurg. Published online November 1, 2019. doi:10.3171/2019.8.JNS191256), in which the authors comprehensively analyzed the research productivity of neurosurgical residency applicants. Wadhwa et al. included the publication volume for all neurosurgery interns in 2009, 2011, 2014, 2016, and 2018, and showed that mean publication numbers among interns at the top 40 programs were significantly higher than that of other interns. Similarly, the 2018 National Resident Matching Report "Charting Outcomes in the Match" reported a significant difference in the mean number of scholarly works (abstracts, presentations, and publications) between matched and unmatched neurosurgery residents across the US (18.3 in matched applicants vs 8.9 in unmatched applicants). ${ }^{2}$ The mean number of scholarly works in matched neurosurgery applicants was also easily the highest amongst the matched applicants of all specialties analyzed in the report.

The authors must be congratulated on their excellent work in providing an in-depth analysis of the research output of neurosurgery residency applicants. The results of this study suggest that neurosurgery is one of the more competitive residency specialties and research productivity is an important aspect in determining an applicant's competitiveness. As such, one would expect medical students pursuing neurosurgery to excel in their research endeavors. Healthy competition drives productivity and growth in these students, spurring them to strive to learn and improve. This ensures that future residents are at their maximum potential at the point of entry into the neurosurgery program. Evidently, healthy competition benefits the development of both the individual student and the neurosurgical specialty in general.

However, competitiveness is a double-edged sword. While healthy competition may be a driver of development, increased competitiveness can be an incentive for an individual to get ahead by any and all means necessary. An example of such unhealthy behavior is the misrepresentation of one's research output on one's application. In a recent article by Kistka et al., the authors analyzed all neurosurgery residency applications to Vanderbilt University in 2006 and 2012, and compared the incidence of publication misrepresentation between the 2 years. ${ }^{3}$ They demonstrated a significant increase in the rate of publication misrepresentation among applicants from 2006 to 2012 (33\% vs $45 \%$ ). Prior to the study by Kistka et al., the most recent analysis of neurosurgery residency applications by Cohen-Gadol et al. in 2001-2002 found misrepresentation in only $6 \%$ of all applicants of the Mayo Medical School. ${ }^{4}$ While we acknowledge that a direct comparison cannot be made between the studies due to the heterogeneous study samples comprising applicants of different residency programs, this huge difference suggests a rising trend in misrepresentation and should not be taken lightly.

In a recent editorial, Dr. Heros very nicely outlined the reasons why research output can be so highly valued in today's residency applications, as well as the factors that contribute to scholarly misrepresentation. ${ }^{5}$ Dr. Heros advocates for increased emphasis to be placed on the quality and impact of publications rather than the absolute quantity. We would like to add that providing recognition not just for the amount of research output, but more importantly for the work ethic, learning attitude, and values of a student or applicant (such as integrity and compassion), can also play a critical role in minimizing overemphasis on research numbers. In essence, healthy competition should always be encouraged, but not at the price of intellectual dishonesty.

John J. Y. Zhang
Yong Loo Lin School of Medicine, National University of Singapore,
Singapore
Keng Siang Lee
University of Bristol,
United Kingdom

\section{References}

1. Wadhwa H, Shah SS, Shan J, et al. The neurosurgery applicant's "arms race": analysis of medical student publication in the Neurosurgery Residency Match. J Neurosurg. Published online November 1, 2019. doi:10.3171/2019.8.JNS191256

2. National Resident Matching Program. Charting Outcomes in the Match: U.S. Allopathic Seniors. Characteristics of U.S. Allopathic Seniors Who Matched to Their Preferred Specialty in the 2018 Main Residency Match. National Resident Matching Program; 2018. Accessed April 2, 2020. https://www.nrmp.org/wp-content/uploads/2018/06/ChartingOutcomes-in-the-Match-2018-Seniors.pdf 
3. Kistka HM, Nayeri A, Wang L, et al. Publication misrepresentation among neurosurgery residency applicants: an increasing problem. J Neurosurg. 2016;124:193-198.

4. Cohen-Gadol AA, Koch CA, Raffel C, Spinner RJ. Confirmation of research publications reported by neurological surgery residency applicants. Surg Neurol. 2003;60(4):280-284.

5. Heros RC. Editorial: Misrepresentation among neurosurgery residency applicants. J Neurosurg. 2016;124(1):190-191.

\section{Disclosures}

The authors report no conflict of interest.

\section{Correspondence}

John J. Y. Zhang: zhangjohnjy@gmail.com.

INCLUDE WHEN CITING

Published online May 8, 2020; DOI: 10.3171/2020.2.JNS20409.

\section{Response}

We appreciate the opportunity to respond to the letter published by Zhang and Lee on our article. Zhang and Lee raise the concept of competition as a double-edged sword; while it can drive potential applicants to pursue research endeavors in neurosurgery and maximize their productivity, it can also promote behaviors such as publication misrepresentation.

So, when does healthy competition tip over into the unhealthy? Indeed, our work raises this very question with its findings. There is a discrepancy between the mean number of publications and the mean number of research products disclosed by the "Charting Outcomes in the Match" (ChOM) reports. ${ }^{1}$ This phenomenon may be due to publications that are not peer-reviewed, presentations, and posters. Or perhaps it is associated with the $45 \%$ of neurosurgery residency applicants who engage in research misrepresentation, as reported by Kistka et al. ${ }^{2}$ Regardless, the competition that is driving the increase in mean publications among neurosurgery residency applicants and publication misrepresentation is a symptom of a residency application process that may need to be reformed.

The skyrocketing costs of the residency application cycle have been well documented by blogs and individual reports. Agarwal et al. surveyed the first-year neurosurgery residents in 2016 and found that the mean total expenses were $\$ 10,255 .^{3}$ Most of the associated costs were attributable to interviews. The "arms race" observed in the research products produced by residency applicants is similarly reflected in the number of applications submitted. According to the $2018 \mathrm{ChOM}$ report, the mean number of contiguous ranks for an applicant who matched into a neurosurgery residency was 16.4, which was increased from 13.5 in 2009. ${ }^{1,4}$ In 2018, 16 contiguous ranks will provide an applicant with an approximately $90 \%$ chance of matching. Yet with the high cost associated with a failure to match and potential re-application, many applicants will apply to far more than 16 programs.

This phenomenon is particularly important in light of recent news from the National Board of Medical Examiners (NBME) announcing that the Step 1 examination will transition to pass/fail grading no earlier than January 2022. At this time, it is difficult to speculate how this transition will impact how residency programs evaluate applications. However, it is reasonable to assume that the importance of other components of the application, including research experience, will increase. As the emphasis in neurosurgery residency application evaluation changes, so too will the concerted effort of applicants. Without a standardized method by which to distinguish abstracts or presentations from peer-reviewed publications, residency programs may find it increasingly difficult to gauge the quality of a candidate's research accomplishments. Of note, the change introduced by the NBME is unlikely to decrease stress for the residency applicant. So long as applicants are allowed to apply to as many programs as they desire, residency programs will need a method by which to judge the quality of hundreds of applications. Thus, the applicant's "arms race" continues.

\section{Harsh Wadhwa, BS Sumedh S. Shah, BS Manish K. Aghi, MD, PhD \\ University of California, San Francisco, CA}

\section{References}

1. National Resident Matching Program. Charting Outcomes in the Match: U.S. Allopathic Seniors. Characteristics of U.S. Allopathic Seniors Who Matched to Their Preferred Specialty in the 2018 Main Residency Match. National Resident Matching Program; 2018. Accessed April 2, 2020. http://www.nrmp.org/wp-content/uploads/2018/06/ChartingOutcomes-in-the-Match-2018-Seniors.pdf

2. Kistka HM, Nayeri A, Wang L, et al. Publication misrepresentation among neurosurgery residency applicants: an increasing problem. J Neurosurg. 2016;124:193-198.

3. Agarwal N, Choi PA, Okonkwo DO, et al. Financial burden associated with the residency match in neurological surgery. J Neurosurg. 2017;126:184-190.

4. National Resident Matching Program. Charting Outcomes in the Match. Characteristics of Applicants Who Matched to Their Preferred Specialty in the 2009 Main Residency Match. National Resident Matching Program; 2009.

Accessed April 2, 2020. http://www.nrmp.org/wp-content/ uploads/2013/08/chartingoutcomes2009v3.pdf

\section{INCLUDE WHEN CITING}

Published online May 8, 2020; DOI: 10.3171/2020.2.JNS20416.

CAANS 2020, except where prohibited by US copyright law

\section{Academic rank achievement by gender}

TO THE EDITOR: We read with great interest the article by Dossani et al. ${ }^{1}$ (Dossani RH, Terrell D, Kosty JA, et al. Gender disparities in academic rank achievement in neurosurgery: a critical assessment [published online November 8, 2019]. J Neurosurg. doi:10.3171/2019.8.JNS191219). The article focuses on neurosurgeons in the US and explores whether gender, particularly being female, is an impediment to progressing through academic ranks. In the raw data, 12 of 48 neurosurgical units have no females on faculty, a further 12 have a single woman, and an addition- 
al 9 have 2 females on faculty. Overall, there are 973 faculty members, of whom 886 are male and 87 are female.

The key analysis of the paper is a logistic regression of academic rank achievement by gender, adjusting for each particular neurosurgical unit, faculty level, years in practice, h-index, and number of papers. From this analysis the authors conclude that if a woman 1) is employed at the same institution, 2) reaches the same faculty level, 3) has the same number of years in practice, 4) has the same h-index, and 5) has the same number of papers as her male colleagues, then there is no evidence to support the conclusion that she will be disadvantaged on the basis of her gender. This is both a misleading and incorrect conclusion. It is misleading because items 1-5 are all points at which gender disadvantage occurs..$^{2-10}$ It is incorrect because, in the terminology of causal inference, the authors have treated mediators (or effect modifiers) as if they are confounders.

Figure 1 sets out two alternative causal diagrams for the relationships between gender, academic rank, and the covariates. The upper part of Fig. 1 depicts what Dossani et al. describe in their paper. Gender is the exposure of interest, academic rank is the outcome, and number of papers is a confounder. The direction of the arrows shows the direction of influence, so number of papers is considered a common cause of both differences in gender and differences in academic rank.

The lower part of Fig. 1 displays the true causal diagram. Here the causal effect of gender on academic rank achievement passes through the number of papers published, institution, $\mathrm{PhD}$ attainment, and so on. In this account, gender biases reduce females' opportunities for research, reducing their number of published papers and, in turn, reducing their prospects for promotion (which is, in

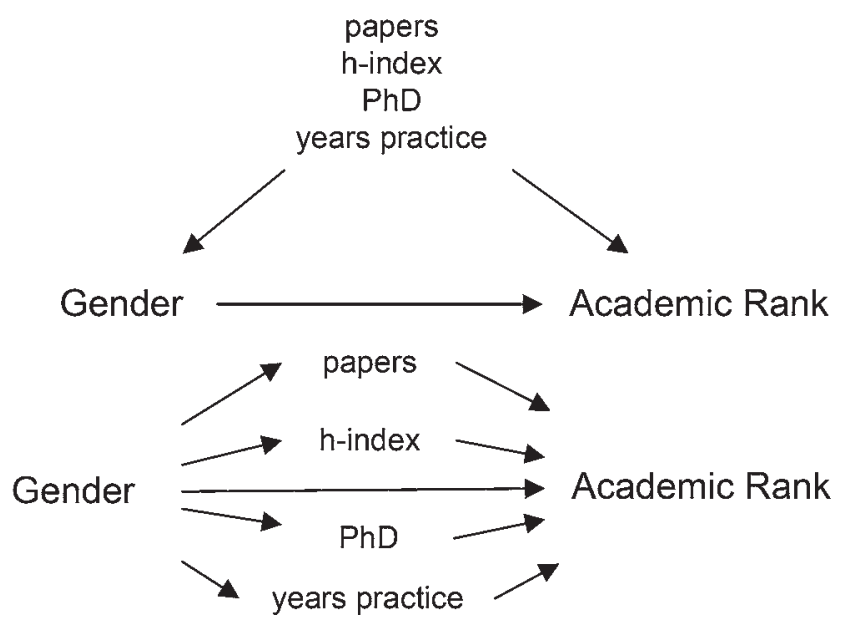

FIG. 1. Illustration of two ways of understanding the relationship among gender, academic rank, and a series of covariates (number of published papers, h-index, PhD completion, and years in practice). The upper diagram shows the relationship reported by Dossani et al., in which the covariates are confounders of the association between gender and academic rank achievement and are not on the causal pathway. The lower diagram depicts the more plausible causal relationship, whereby the covariates sit on the causal pathway and mediate the association between gender and academic rank. every institution, at least partly based on publication record).

Adjusting for mediators in a logistic regression model (as reported in this study) falsely reduces the apparent causal effect of gender on academic achievement by discarding any of the causal effect operating through the mediator. Appropriate mediation analyses exist and are available in the major statistical packages.

It matters a great deal whether we tell female doctors and trainees, "if you work hard, your gender alone will not disadvantage you" or "if you work hard and struggle past all other forms of gender discrimination, ultimately your gender will not disadvantage you." The first is hopeful, the second is cruel. Unfortunately, the study by Dossani et al. has reported the second scenario as if it were the first.

Bradley R. Crammond, MBiostat, PhD Melbourne School of Population and Global Health, University of Melbourne, Parkville, Australia

Neela Janakiramanan, MBBS, MPH Eastern Health, Melbourne, Australia

Mario Ganau, MD, PhD

Oxford University Hospitals NHS Trust, Oxford, United Kingdom

Deborah Verran, MBChB, MHSM

Royal Prince Alfred Hospital, Sydney, Australia

Laura Lippa, MD

Ospedali Riuniti di Livorno, Italy

Jonathan Braman, MD

University of Minnesota Medical School, Minneapolis, MN

Katrin Rabiei, MD, PhD

Institute of Neuroscience and Physiology, Sahlgrenska Academy, Gothenburg, Sweden

\section{References}

1. Dossani RH, Terrell D, Kosty JA, et al. Gender disparities in academic rank achievement in neurosurgery: a critical assessment [published online November 8, 2019]. J Neurosurg. doi:10.3171/2019.8.JNS191219

2. Abelson JS, Chartrand G, Moo TA, et al. The climb to break the glass ceiling in surgery: trends in women progressing from medical school to surgical training and academic leadership from 1994 to 2015. Am J Surg. 2016;212:566-572. e561.

3. Carr PL, Gunn CM, Kaplan SA, et al. Inadequate progress for women in academic medicine: findings from the National Faculty Study. J Womens Health (Larchmt). 2015;24:190199.

4. McDermott M, Gelb DJ, Wilson K, et al. Sex differences in academic rank and publication rate at top-ranked US neurology programs. JAMA Neurol. 2018;75:956-961.

5. McKinsey \& Company. Women in the Workplace 2019. Accessed March 9, 2020. https://womenintheworkplace.com

6. Odell T, Toor H, Takayanagi A, et al. Gender disparity in academic neurosurgery. Cureus. 2019;11:e4628.

7. Seemann NM, Webster F, Holden HA, et al. Women in academic surgery: why is the playing field still not level? Am J Surg. 2016;211:343-349.

8. Sexton KW, Hocking KM, Wise E, et al. Women in academic surgery: the pipeline is busted. J Surg Educ. 2012;69:84-90.

9. van den Besselaar P, Sandstrom U. Gender differences in research performance and its impact on careers: a longitudinal case study. Scientometrics. 2016;106:143-162. 
10. Wenneras C, Wold A. Nepotism and sexism in peer-review. Nature. 1997;387:341-343.

\section{Disclosures}

The authors report no conflict of interest.

\section{Correspondence}

Bradley R.Crammond: brad.crammond@unimelb.edu.au.

\section{INCLUDE WHEN CITING}

Published online March 27, 2020; DOI: 10.3171/2020.1.JNS2052.

\section{Response}

We appreciate the interesting commentary of Dr. Crammond and colleagues regarding our recent publication. The primary point the authors make is that female gender affects not only rank attainment, but also the ability to achieve milestones that are independent predictors of rank attainment. They assert that because gender affects the other predictors of rank attainment in our linear regression model, these factors are effect modifiers, not confounders, and linear regression is an inappropriate test for analysis. This is a very interesting assertion, but difficult to prove.

The authors state that there is gender disadvantage in 1) the hiring of women at an academic center, 2) the number of years in practice, 3) h-index, and 4) the number of papers produced. They cite as evidence several papers from academic surgery, but only one, by Odell et al., is specific to neurosurgery. ${ }^{1}$ The findings of Odell et al. are similar to ours, which is that there are more women in assistant and associate professor roles than full professor roles. Using the neurosurgical literature, we will examine the available data regarding the effect of gender on each of the variables in our model below.

Assertion: There is a gender disadvantage in the hiring of women at an academic center.

Response: This is unlikely to be the case, as the percentage of women at the assistant and associate professor-level ranks $(12 \%)$ is comparable to the percentage of women entering neurosurgery over the past 20 years $(12 \%){ }^{2}$ Given that training takes 7 years, and that as of 2009 there was a $17 \%$ attrition rate for women in neurosurgical residency, there may even be a slightly higher percentage of women in academic neurosurgery than in the graduating classes. ${ }^{2}$ For the 2000-2007 match years, gender actually was an independent negative predictor of matching into a neurosurgery program; however, women had significantly lower step 1 scores than their male counterparts. ${ }^{3}$ It is unclear whether this effect is still present 13 years later.

Given the large discrepancy between the numbers of men and women entering and completing neurosurgical residency, it is likely that internal factors (i.e., the decisions made by women) are as, if not more, important as external factors such as gender bias. Neurosurgery may not appeal to women or end up being a good career fit for several reasons, including the unique challenges it presents to bearing and raising children. This should not be seen as a failure of women to achieve career goals, but rather an active decision to pursue a different career more consistent with life goals.

Assertion: There is a gender disadvantage to the number of years in practice.
Response: Although there is a higher attrition rate in residency for women than men, ${ }^{2}$ to our knowledge, there is no evidence that the length of careers for women in academic neurosurgery is shorter than the length of those for men. Because women have been increasingly entering neurosurgery over the past 20 years, on average, the years in practice for women is less than that for men as a reflection of this trend.

Assertion: There is a gender disadvantage to having a higher h-index or greater number of papers published.

Response: Given that most peer-reviewed journals utilize blinded reviews, it is highly unlikely that female gender is a disadvantage to having one's work accepted to a journal. Indeed, several authors have compared the academic productivity of neurosurgeons and found no significant gender gap after controlling for rank and years in practice $^{4-6}$

While female gender may interact with each of these variables, there are little data from the neurosurgical literature to back this assertion. Women may continue to face discrimination in academic neurosurgery that is manifested in ways we have not and cannot account for as our analysis was limited to the variables described above. We do feel, however, that the increasing number of women in the field has worked to reduce biases that were likely more pronounced in the past. We are heartened that our data support an optimistic message, which is that gender may not inherently be a negative predictor of career advancement in academic neurosurgery.

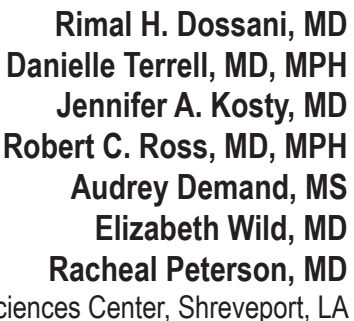

Louisiana State University Health Sciences Center, Shreveport, LA

Laura B. Ngwenya, MD, PhD

University of Cincinnati Medical Center, Cincinnati, $\mathrm{OH}$

Deborah L. Benzil, MD

Cleveland Clinic Foundation, Cleveland, $\mathrm{OH}$

Christina Notarianni, MD

Louisiana State University Health Sciences Center, Shreveport, LA

\section{References}

1. Odell T, Toor H, Takayanagi A, et al. Gender disparity in academic neurosurgery. Cureus. 2019;11:e4628.

2. Renfrow JJ, Rodriguez A, Liu A, et al. Positive trends in neurosurgery enrollment and attrition: analysis of the 2000-2009 female neurosurgery resident cohort. J Neurosurg. 2016;124:834-839.

3. Durham SR, Donaldson K, Grady MS, Benzil DL. Analysis of the 1990-2007 neurosurgery residency match: does applicant gender affect neurosurgery match outcome? J Neurosurg. 2018;129:282-289.

4. Khan NR, Thompson CJ, Taylor DR, et al. An analysis of publication productivity for 1225 academic neurosurgeons and 99 departments in the United States. J Neurosurg. 2014;120:746-755. 
5. Reddy V, Gupta A, White MD, et al. Assessment of the $\mathrm{NIH}$-supported relative citation ratio as a measure of research productivity among 1687 academic neurological surgeons [published online January 31, 2020]. J Neurosurg. doi:10.3171/2019.11.JNS192679

6. Tomei KL, Nahass MM, Husain Q, et al. A gender-based comparison of academic rank and scholarly productivity in academic neurological surgery. J Clin Neurosci. 2014;21:1102-1105.

\section{INCLUDE WHEN CITING}

Published online March 27, 2020; DOI: 10.3171/2020.2.JNS20180.

CAANS 2020, except where prohibited by US copyright law

\section{Cognitive performance of patients with low-grade glioma}

TO THE EDITOR: I read with interest the article by Rijnen et al. ${ }^{1}$ (Rijnen SJM, Kaya G, Gehring K, et al. Cognitive functioning in patients with low-grade glioma: effects of hemispheric tumor location and surgical procedure [published online November 15, 2019]. J Neurosurg. doi:10.3171/2019.8.JNS191667). Dr. Rijnen and colleagues "evaluated the cognitive performance of patients with lowgrade glioma (LGG) before and after surgery, and specifically investigated 1) the effects of hemispheric tumor location and 2) the type of surgery (either with or without intraoperative stimulation mapping [ISM])." The study was funded by the Netherlands Organisation for Health Research and Development, project number 842003007.1,2 It received ethics approval, but a protocol number is not provided in this journal. ${ }^{1}$ This contrasts to a different study that was funded under the same grant, ${ }^{2,3}$ which provides a link to an abstract of an approved study protocol (NL41351.008.12) in the Netherlands Trial Register. ${ }^{4}$ Investigating the effects of hemispheric tumor location and type of surgery was not described in the protocol objectives as provided by the funder ${ }^{2}$ or in the objectives listed in the Netherlands Trial Register. ${ }^{4}$ Protocol objective 2 was "to develop models based on presurgical sociodemographic, clinical, imaging, and (neuro)psychological variables that predict cognitive functioning one year after surgery" (in patients with glioma and meningiomas, F.V.). ${ }^{4}$ The prognostic models were supposed to be developed with machine learning techniques. ${ }^{2}$ As far as I can see, no clinical prediction models have been developed for prediction of cognitive function after surgery of meningiomas ${ }^{3}$ or gliomas. ${ }^{1,2}$ While work by Dr. Rijnen and colleagues has been funded ${ }^{2}$ and ethically approved, I noticed several discrepancies between the preapproved study objectives and the current paper ${ }^{1}$ and other work by members of the same group, ${ }^{3}$ both with regard to objectives and methods. Given that I do not have access to the full study protocol(s) or grant proposals, I would appreciate if Dr. Rijnen and colleagues could help to better delineate the underlying links between the project goals and report as published on their funder's website, ${ }^{2}$ the ethically approved study protocol(s), ${ }^{4}$ and, if applicable, clarify and expand on any potential deviations. ${ }^{1,3}$
Frank de Vries, PharmD, PhD

Maastricht University Medical Centre, Maastricht, The Netherlands Utrecht Institute for Pharmaceutical Sciences, Utrecht, The Netherlands

\section{References}

1. Rijnen SJM, Kaya G, Gehring K, et al. Cognitive functioning in patients with low-grade glioma: effects of hemispheric tumor location and surgical procedure [published online November 15, 2019]. J Neurosurg. doi:10.3171/2019.8.JNS191667

2. Netherlands Organisation for Health Research and Development. Cognitive deficits in brain tumor patients after neurosurgery: incidence, severity and prediction of outcome. ZonMw project number 842003007 . Accessed March 27, 2020. https://www.zonmw.nl/nl/onderzoekresultaten/kwaliteit-van-zorg/medisch-specialistische-zorg/ programmas/project-detail/topzorg/cognitive-deficits-inbrain-tumor-patients-after-neurosurgery-incidence-severityand-prediction-of/

3. Rijnen SJM, Meskal I, Bakker M, et al. Cognitive outcomes in meningioma patients undergoing surgery: individual changes over time and predictors of late cognitive functioning. Neuro Oncol. 2019;21(7):911-922.

4. Netherlands Trial Register. Trial NL5063. Cognitive deficits in brain tumor patients after neurosurgery: incidence, severity and prediction of outcome. Accessed March 27, 2020. https://www.trialregister.nl/trial/5063

\section{Disclosures}

The author co-supervises two $\mathrm{PhD}$ students who are employed by F. Hoffmann la Roche (Basel, Switzerland, and Welwyn Garden City, United Kingdom). The topics of their PhDs are not related to this letter, and the author has not received any fees or reimbursements for this.

\section{Correspondence}

Frank de Vries: frank.de.vries@mumc.nl.

\section{INCLUDE WHEN CITING}

Published online April 24, 2020; DOI: 10.3171/2020.1.JNS2097.

\section{Response}

We thank the editor for the invitation to reply to the comments of Prof. de Vries on our article. Professor de Vries suggests that the objectives of our study have not been described in the study protocol. The objectives to which Prof. de Vries refers are described on the website of the Netherlands Organisation for Health Research and Management (ZonMw) ${ }^{1}$ and the Netherlands Trial Register. ${ }^{2}$ These objectives are part of an overarching research protocol that was approved by the Medical Ethical Committee Brabant, the Netherlands (file number NL41351.008.12), in which, among others, the following objectives are explicitly stated: "to describe the incidence and severity of cognitive impairments in these patients before, and 3 months after treatment" and "to identify the predictors (sociodemographic, clinical, imaging, and (neuro)psychological variables) of objective cognitive functioning of all patients who undergo neurosurgery." As the aim of the current study was "[to evaluate] the cognitive performance of patients with low-grade glioma (LGG) before and after surgery, and specifically [to investigate] 1) the effects of hemispheric tumor location and 2) the type 
of surgery," the study is covered by the overarching project's protocol without any concerns.

In addition, Prof. de Vries remarks that a protocol number is not provided in the published article. This is correct, yet the protocol number NL41351.008.12 was included in all submitted versions of our manuscript. In the final stage of the production, the number was removed following the publishing policies of the Journal of Neurosurgery.

\section{Sophie J. M. Rijnen, PhD1 Karin Gehring, $\mathrm{PhD}^{1,2}$ Margriet M. Sitskoorn, PhD1 Geert-Jan M. Rutten, MD, PhD² \\ ${ }^{1}$ Tilburg University, Tilburg, The Netherlands ${ }^{2}$ Elisabeth-TweeSteden Hospital, Tilburg, The Netherlands}

\section{Editor's Note}

When authors submit manuscripts to the Journal of Neurosurgery Publishing Group (JNSPG) that describe clinical studies, they are required to submit an authorization number from their local internal review board/medical ethics committee to confirm that the study protocol was reviewed and approved. As a policy, the JNSPG does not include this internal number in the published article but does require an author statement in the manuscript that the study was approved by this committee and that all participants provided written informed consent. Such a statement was included in the article by Dr. Rijnen and colleagues.

\section{James T. Rutka, MD, PhD Editor-in-Chief Journal of Neurosurgery Publishing Group, Charlottesville, VA}

\section{References}

1. Netherlands Organisation for Health Research and Development. Cognitive deficits in brain tumor patients after neurosurgery: incidence, severity and prediction of outcome. ZonMw project number 842003007 . Accessed March 27, 2020. https://www.zonmw.nl/nl/onderzoek-resultaten/kwaliteit-van-zorg/medisch-specialistische-zorg/programmas/project-detail/topzorg/cognitive-deficits-in-brain-tumor-patientsafter-neurosurgery-incidence-severity-and-prediction-of/

2. Netherlands Trial Register. Trial NL5063. Cognitive deficits in brain tumor patients after neurosurgery: incidence, severity and prediction of outcome. Accessed March 27, 2020. https://www.trialregister.nl/trial/5063

\section{INCLUDE WHEN CITING}

Published online April 24, 2020; DOI: 10.3171/2020.3.JNS20179.

CAANS 2020, except where prohibited by US copyright law

\section{Pupillometry and neurotrauma: point-of-care technologies versus point-of-care techniques}

TO THE EDITOR: We read with interest the article by Joseph et al.' (Joseph JR, Swallow JS, Willsey K, et al. Pupillary changes after clinically asymptomatic highacceleration head impacts in high school football athletes [published online November 26, 2019]. J Neurosurg. doi:10.3171/2019.7.JNS191272).

The role of point-of-care testing has been increasingly recognized as a way to improve outcomes in healthcare. The available data on point-of-care testing are limited and suggest that point-of-care testing is costlier than traditional laboratory investigations. ${ }^{2}$ In contrast to laboratory investigations, in the present article the authors explore the role of the clinical parameter "measurable changes in pupil response in clinically asymptomatic high school football athletes following high-acceleration head impacts"1 as an alternative to point-of-care technologies. We congratulate the authors for their excellent work, which expands the concept of clinical examination using clinical markers (in contrast to laboratory biomarkers) as highly cost-effective, noninvasive, and efficient tools to monitor the injured in a real-life scenario.

To further add to the comprehensive discussion presented by Joseph et al., we would like to add that the innervation of the eyes represents a complex balance of sympathetic and parasympathetic functions. This balance could be altered by many mechanisms, including intoxication, trauma to the head, direct trauma to the eyes, etc. For athletes participating in contact sports, it has been reported that up to $90 \%$ will experience a head injury, depending on the type of sport, which makes the topic of Joseph et al.'s article crucial for athletes, parents, trainers, lawyers, etc.

The information presented by these authors is important for two reasons. First, regarding the baseline conditions of pupil constriction velocity, latency to constriction, maximum size, and percentage change, in the reported study maximum constriction velocity and dilation velocity were recorded in controls before the events of head trauma and serve as baseline data. In addition, pupil dilation velocity, percentage change in pupil size, and maximum constriction velocity parameters showed statically significant alterations after a mild traumatic brain injury (mTBI), findings that have already been confirmed by Ciuffreda et al. ${ }^{3}$ However, the changes of these parameters could not be interpreted to be due only to anatomical changes or neuron pathway dysfunction. As already described by Shetty et al., ${ }^{4}$ about $27 \%$ of the patients after an mTBI develop white matter changes. Of the neuropsychological assessments used, the 5- and 6-Digit Backward Recall, the modified Balance Error Scoring System (BESS), and the Immediate 5-Word Recall significantly improved longitudinally in mTBI subjects and facilitated differentiation between the mTBI subjects and controls. Female subjects tend to score more points on symptom severity scores, as well as older patients ( $\geq 25$ years of age) and patients with a history of psychiatric disorder. The second reason for the importance of this study is that it could serve as a basis for upgrading the graduated return-to-play protocol, which is already in use but should include the application of clinical findings. The quantitative pupillometry method used by Joseph et al. offers a unified, objective method to determine the parameters of the pupil in relation to mTBI.

Combining neuroimaging, biomarkers, neurocognitive evaluation, quantitative pupillometry, and clinical findings 
is needed to increase our understanding of the neurophysiological correlations between these variables. In addition, anatomical changes are often clinically silent, and uncertainty remains regarding which neuron pathway dysfunctions are without anatomical correlations. We believe that the present study will provide a road map to conduct further research and elucidate how clinical techniques can be used as reliable outcome markers as well as reliable clinical biomarkers that can be incorporated into an alternative point-of-care method to identify and follow up cases of mTBI injuries with neurological sequelae.

\section{Orlin Pavlov, MD \\ Fulda Clinic, Fulda, Germany}

Rafael Martinez-Perez, MD, PhD

The Ohio State University, Columbus, $\mathrm{OH}$

Amit Agrawal, MD, MCH

Narayana Medical College and Hospital, Nellore, Andhra Pradesh, India

Ezequiel Garcia-Ballestas, MD

Luis Rafael Moscote-Salazar, MD

Center for Biomedical Research (CIB), Faculty of Medicine, University of Cartagena, Colombia

\section{References}

1. Joseph JR, Swallow JS, Willsey K, et al. Pupillary changes after clinically asymptomatic high-acceleration head impacts in high school football athletes [published online November 26, 2019]. J Neurosurg. doi:10.3171/2019.7.JNS191272

2. Gutierres SL, Welty TE. Point-of-care testing: an introduction. Ann Pharmacother. 2004;38(1):119-125.

3. Ciuffreda KJ, Joshi NR, Truong JQ. Understanding the effects of mild traumatic brain injury on the pupillary light reflex. Concussion. 2017;2(3):CNC36.
4. Shetty T, Nguyen JT, Cogsil T, et al. Clinical findings in a multicenter MRI study of mild TBI. Front Neurol. 2018;9:836.

\section{Disclosures}

The authors report no conflict of interest.

\section{Correspondence}

Luis Rafael Moscote-Salazar: rafaelmoscote21@gmail.com.

\section{INCLUDE WHEN CITING}

Published online April 24, 2020; DOI: 10.3171/2020.1.JNS193487.

\section{Response}

We appreciate the interest in our research expressed by Pavlov et al., and their comments are well taken. The physiology of the pupillary light reflex is highly complex, making it difficult to definitely state what the root cause of changes may be. The variable activation of the autonomic nervous system during sporting activity is particularly difficult to control for. However, we do believe that the results presented in our article are promising, and we agree that objective clinical measures such as quantitative pupillometry and eye tracking can potentially enhance return-toplay protocols.

Jacob R. Joseph, MD

University of Michigan, Ann Arbor, Ml

INCLUDE WHEN CITING

Published online April 24, 2020; DOI: 10.3171/2020.2.JNS20213.

๑AANS 2020, except where prohibited by US copyright law 